\title{
Synthesis and Structural Characterization of 1,4-Diazepines
}

Related to Curcumin

\section{Ana Andrade ${ }^{a}$, Dionisia Sanz ${ }^{a, *}$, Rosa M. Claramunt ${ }^{a}$ and José Elguero ${ }^{b}$}

aDepartamento de Química Orgánica y Bio-Orgánica, Facultad de Ciencias, UNED, Paseo de Senda del Rey 9, E-28040 Madrid, Spain

' Instituto de Química Médica, Centro de Química Orgánica Manuel Lora-Tamayo, CSIC, Juan de la Cierva 3, E-28006 Madrid, Spain

* Corresponding author. Tel.: + 34913987331; fax: + 34913988372.

E-mails: dsanz@ccia.uned.es

Abstract: The reactivity of (E)-5-(4-hydroxy-3-methoxyphenyl)-1-phenylpent-4-ene-1,3-dione with ethylenediamine has been studied in different experimental conditions. Two dihydro-1,4-diazepines have been obtained and fully characterized by $1 \mathrm{D}$ and $2 \mathrm{D}$ multinuclear NMR spectroscopy and HRMS.

Keywords: 1,4-diazepines, curcumin, ${ }^{1} \mathrm{H},{ }^{13} \mathrm{C},{ }^{15} \mathrm{~N}$ NMR

\section{Introduction}

The interest in curcumin $(1)^{1}$ has prompted many studies on the synthesis, characterization and biological properties of compounds resulting from structural modifications, i.e. the hemicurcuminoids, obtained by replacement of one styryl (2-methoxy-4-vinylphenol) branch of curcumin by a simpler group. ${ }^{2}$ Curcumin or [(1E,6E)-1,7-bis(4-hydroxy-3-methoxyphenyl)hepta-1,6diene-3,5-dione], is a $\beta$-diketone that owing to its symmetry has only two tautomers, enol 1a and keto $\mathbf{1 b}$ (Fig. 1), the third one being identical to $1 \mathrm{a}$.<smiles>COc1cc(/C=C/C(=O)CC(=O)/C=C/c2ccc(/C=C/C(=O)/C=C(O)/C=C/c3ccc(O)c(OC)c3)c(OC)c2)ccc1O</smiles>

Figure 1. Curcumin tautomers.

Curcumin and hemicurcuminoids have different reactive functional groups, ${ }^{1 d} \beta$-diketo and $\alpha, \beta$-unsaturated keto, that can participate in nucleophilic addition reactions to yield pyrazoles, ${ }^{3}$ isoxazoles, ${ }^{4}$ and other heterocyclic systems. ${ }^{5}$ The phenolic group can react with fatty acids, amino acids, etc. to enhance bioavailability. ${ }^{6}$ 


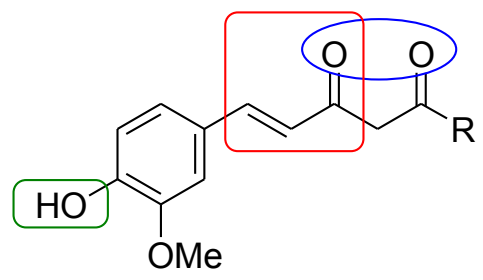

Figure 2. Reactive functional groups of hemicurcuminoids.

\section{Results and discussion}

The reactivity of curcumin with 1,2-ethylenediamine has already been studied by two research groups. ${ }^{7,8}$ In this work we present the reaction of the hemicurcuminoid (E)-5-(4-hydroxy-3methoxyphenyl)-1-phenylpent-4-ene-1,3-dione (2), ${ }^{2 a}$ with ethylenediamine in two different media. In acetic acid it reacts as a $\beta$-dicarbonyl compound leading to (4E,6Z)-7-((E)-3,4-dimethoxystyryl)5-phenyl-2,3-dihydro-1H-1,4-diazepine (3). Differently, in methanol it reacts as a $\alpha, \beta$-unsaturated ketone to give (Z)-2-(7-(4-hydroxy-3-methoxyphenyl)-1,4-diazepan-5-ylidene)-1-phenylethanone (4), as shown in Scheme 1. Yields were almost quantitative in both cases, but after purification 3 [recrystallized from $\mathrm{H}_{2} \mathrm{O} / \mathrm{EtOH}$; HRMS (ESI) m/z calcd for $\mathrm{C}_{20} \mathrm{H}_{20} \mathrm{~N}_{2} \mathrm{O}_{2}$ : 320.15, found: 321.15 $(\mathrm{M}+\mathrm{H})^{+}$] was obtained in a $60 \%$ and 4 [chromatographed over Silica gel and recrystallized from EtOH; HRMS (ESI) m/z calcd for $\mathrm{C}_{20} \mathrm{H}_{22} \mathrm{~N}_{2} \mathrm{O}_{3}: 338.16$, found: $339.17(\mathrm{M}+\mathrm{H})^{+}$] in a $35 \%$.

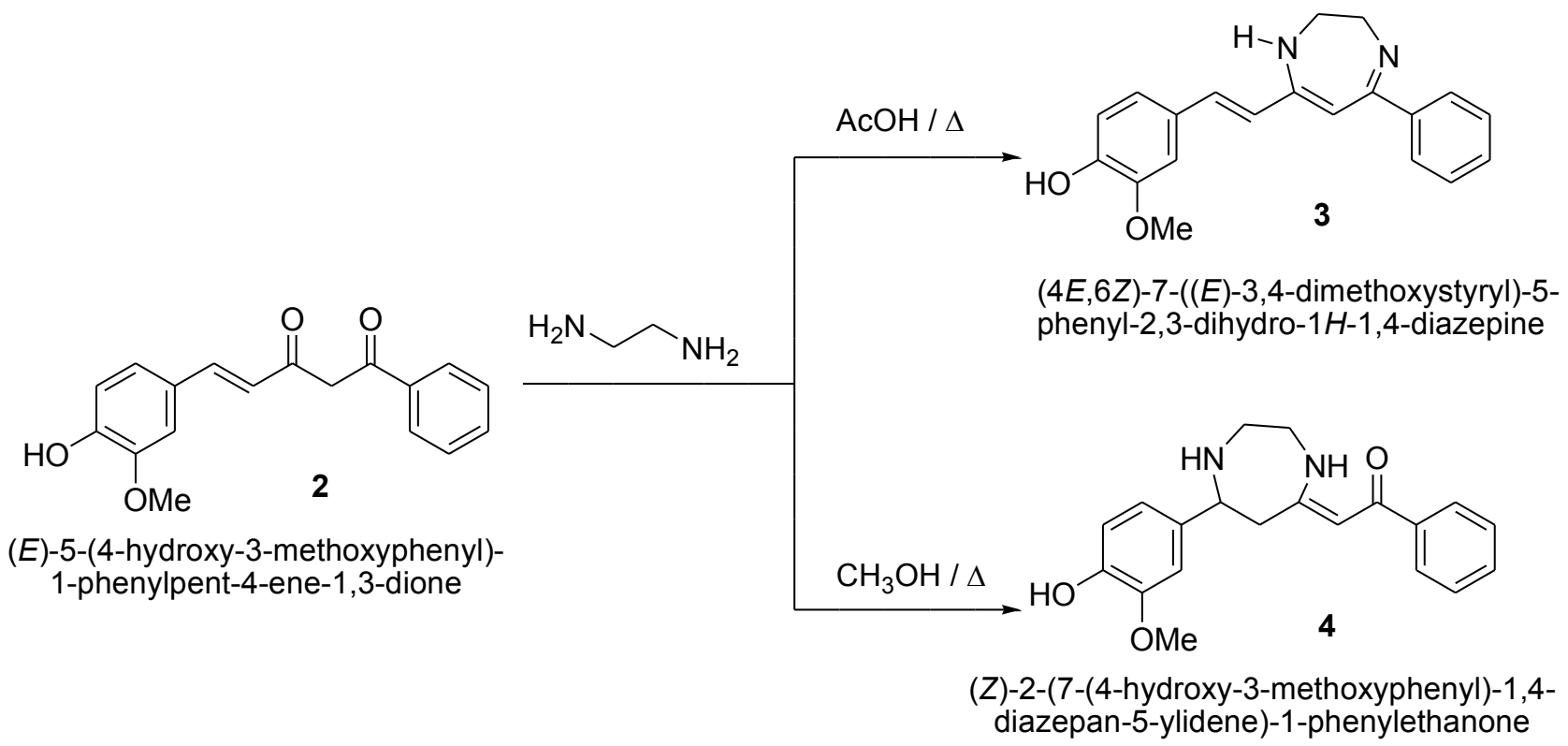

Scheme 1

In Tables 1 and 2 are gathered the most significant ${ }^{1} \mathrm{H},{ }^{13} \mathrm{C}$ and ${ }^{15} \mathrm{~N}$ NMR experimental data chemical shifts ( $\delta$ in $\mathrm{ppm}$ ) and coupling constants ( $J \mathrm{in} \mathrm{Hz}$ ). The attribution of the chemical shifts was based on the multiplicity of the signals as well as on the cross-peaks observed in the $\left({ }^{1} \mathrm{H}-{ }^{13} \mathrm{C}\right)$ gs-HMQC and $\left({ }^{1} \mathrm{H}-{ }^{13} \mathrm{C}\right)$ gs-HMBC bidimensional spectra. 
Table 1. NMR data, chemical shifts $(\delta, \mathrm{ppm})$ and coupling constants $(\mathrm{J}, \mathrm{Hz})$, of compound 3

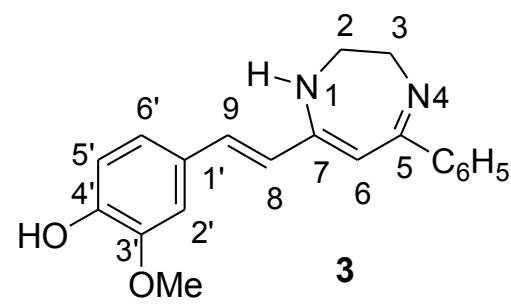

\begin{tabular}{|c|c|c|c|c|c|c|}
\hline & \multicolumn{3}{|c|}{ HMPA-d ${ }_{18}$ at $340 \mathrm{~K}$} & \multicolumn{3}{|c|}{ TFA at $300 \mathrm{~K}$} \\
\hline Nuclei & $\delta$ & $\begin{array}{l}\text { gs-HMQC } \\
\text { correlation }\end{array}$ & $\begin{array}{l}\text { gs-HMBC } \\
\text { correlation }\end{array}$ & $\delta$ & $\begin{array}{l}\text { gs-HMQC } \\
\text { correlation }\end{array}$ & $\begin{array}{l}\text { gs-HMBC } \\
\text { correlation }\end{array}$ \\
\hline 1-NH & n.o. & -- & -- & $-249.5^{*}$ & $9.59(\mathrm{NH})$ & $5.70(\mathrm{H} 6)$ \\
\hline $2-\mathrm{CH}_{2}$ & 50.3 & 3.49 & -- & $48.0^{*}$ & a & \\
\hline $3-\mathrm{CH}_{2}$ & 55.6 & 3.88 & & $47.7^{*}$ & a & \\
\hline $4-N$ & n.o. & --- & -- & $-251.8^{*}$ & $9.86(\mathrm{NH})$ & $5.70(\mathrm{H} 6)$ \\
\hline $5-C$ & $165.3(\mathrm{br})$ & & & 166.8 & & $7.50(\mathrm{Ho})$ \\
\hline $6-C$ & 93.1 & $5.33(\mathrm{H} 6)$ & $6.63(\mathrm{H} 8)$ & $\begin{array}{l}88.8 \\
1 \mathrm{~J}=163.0\end{array}$ & $5.70(\mathrm{H} 6)$ & $6.62(\mathrm{H} 8)$ \\
\hline 7-C & 151.7 (br) & & & 163.3 & & $7.29(\mathrm{H} 9)$ \\
\hline $8-C$ & $125.6(\mathrm{br})$ & $6.56(\mathrm{H} 8)$ & & $\begin{array}{l}119.8 \\
1 \mathrm{~J}=158.8\end{array}$ & $6.62(\mathrm{H} 8)$ & $5.70(\mathrm{H} 6)$ \\
\hline $9-C$ & 131.8 & $7.12(\mathrm{H} 9)$ & $\begin{array}{l}6.91\left(\mathrm{H}^{\prime}\right) \\
7.00\left(\mathrm{H} 2^{\prime}\right)\end{array}$ & $\begin{array}{l}140.5 \\
{ }^{1} J=152.3\end{array}$ & $7.29(\mathrm{H} 9)$ & $7.02\left(\mathrm{H} 6{ }^{\prime}\right)$ \\
\hline $\mathrm{Ci}$ & $143.4(\mathrm{br})$ & & $\begin{array}{l}7.33 \\
(\mathrm{Hm} / \mathrm{p})\end{array}$ & 135.1 & & $\begin{array}{l}5.70(\mathrm{H} 6) \\
7.40(\mathrm{Hm})\end{array}$ \\
\hline C1' & 128.1 & & $\begin{array}{l}6.63(\mathrm{H} 8) \\
6.86\left(\mathrm{H}^{\prime}\right)\end{array}$ & $\begin{array}{l}127.5 \\
3 J=3 J=6.5\end{array}$ & & $\begin{array}{l}6.62(\mathrm{H} 8) \\
6.88\left(\mathrm{H}^{\prime}\right)\end{array}$ \\
\hline C2' & 113.0 & $7.00\left(\mathrm{H}^{\prime}\right)$ & & $\begin{array}{l}109.9 \\
{ }^{1} J=157.7 \\
{ }^{3} J={ }^{3} J=5.9\end{array}$ & $7.04\left(\mathrm{H}^{\prime}\right)$ & $\begin{array}{l}7.02\left(\mathrm{H}^{\prime}\right) \\
7.27(\mathrm{C} 9)\end{array}$ \\
\hline C3' & 148.9 & & $\begin{array}{l}3.80\left(\mathrm{OCH}_{3}\right) \\
6.86\left(\mathrm{H}^{\prime}\right)\end{array}$ & 146.4 & & $\begin{array}{l}3.80\left(\mathrm{OCH}_{3}\right) \\
6.80\left(\mathrm{H}^{\prime}\right)\end{array}$ \\
\hline $3-\mathrm{OCH}_{3}$ & 56.8 & 3.80 & & $\begin{array}{l}54.8 \\
1 \mathrm{~J}=145.5\end{array}$ & 3.85 & \\
\hline C4' & 150.0 & & $\begin{array}{l}6.91\left(\mathrm{H}^{\prime}\right) \\
7.00\left(\mathrm{H} 2^{\prime}\right)\end{array}$ & 145.7 & & $\begin{array}{l}7.02\left(\mathrm{H}^{\prime}\right) \\
7.04\left(\mathrm{H} 2^{\prime}\right)\end{array}$ \\
\hline C5 & 116.4 & $6.86\left(\mathrm{H}^{\prime}\right)$ & & $\begin{array}{l}114.4 \\
1 \mathrm{~J}=162.4\end{array}$ & $6.88\left(\mathrm{H}^{\prime}\right)$ & \\
\hline C6' & 120.9 & $6.91\left(\mathrm{H6}^{\prime}\right)$ & $7.00\left(\mathrm{H}^{\prime}\right)$ & $\begin{array}{l}122.3 \\
1 J=160.7\end{array}$ & $7.02\left(\mathrm{H} 6^{\prime}\right)$ & $\begin{array}{l}7.04\left(\mathrm{H} 2{ }^{\prime}\right) \\
7.29(\mathrm{H} 9)\end{array}$ \\
\hline
\end{tabular}

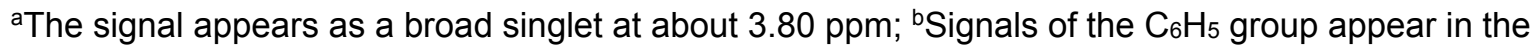
usual range. 
In compound 3 , to assign the $\mathrm{C} 5$ and $\mathrm{C} 7$ chemical shifts we have used the correlation between them with $\mathrm{Ho}$ and $\mathrm{H} 8 / \mathrm{H} 9$, respectively (Figure $3 \mathrm{a}$ ); and the correlation of $\mathrm{C}^{\prime}$ ' with the protons of methoxy group, has permitted to distinguish it from C4' (Figure 3b)

a)

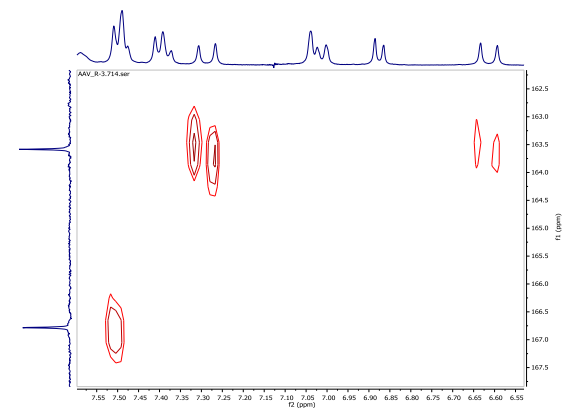

b)

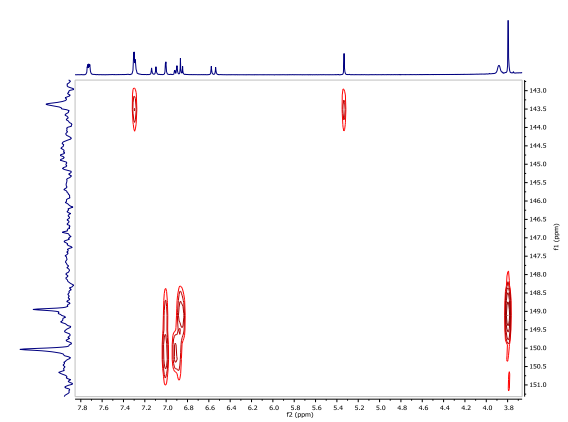

Figura 3. a) $\left({ }^{1} \mathrm{H}-{ }^{13} \mathrm{C}\right)$ gs- $\mathrm{HMBC}$ of $\mathrm{C} 5$ and $\mathrm{C} 7$ in TFA, b) $\left({ }^{1} \mathrm{H}-{ }^{13} \mathrm{C}\right)$ gs- $\mathrm{HMBC}$ of $\mathrm{Ci}, \mathrm{C} 3$ ' and $\mathrm{C} 4$ ' in HMPA-d ${ }_{18}$

Table 4. NMR data, chemical shifts $(\delta, \mathrm{ppm})$ and coupling constants $(\mathrm{J}, \mathrm{Hz})$, of compound 4<smiles>COc1ccc(C2CCCC(=CC(=O)O)C2)cc1</smiles>

\begin{tabular}{|c|c|c|c|c|}
\hline & \multicolumn{2}{|c|}{$\mathrm{CDCl}_{3}$ at $300 \mathrm{~K}$} & \multicolumn{2}{|c|}{ DMSO- $d_{6}$ at $300 \mathrm{~K}$} \\
\hline Nuclei & $\delta$ & $J$ & $\delta$ & $J$ \\
\hline $1-\mathrm{CO}$ & 188.5 & & 186.0 & ${ }^{3} \mathrm{~J}={ }^{3} \mathrm{~J}={ }^{3} \mathrm{~J}=3.9$ \\
\hline $2-\mathrm{CH}$ & 92.0 & $1 \mathrm{~J}=160.8$ & 91.0 & $1 \mathrm{~J}=161.3$ \\
\hline 1'-NH & -318.3 & & -319.9 & \\
\hline 2'- $-\mathrm{CH}_{2}$ & 50.2 & ${ }^{1} \mathrm{~J}=133.1$ & 49.5 & $1 \mathrm{~J}=133.4$ \\
\hline 3'- $-\mathrm{CH}_{2}$ & $46.5^{*}$ & $1 \mathrm{~J}=136.0$ & $46.4^{*}$ & $1 \mathrm{~J}=138.1$ \\
\hline 4'-NH & -264.6 & & -261.1 & \\
\hline $5^{\prime}-C$ & 168.4 & & 168.9 & \\
\hline $6^{\prime}-\mathrm{CH}_{2}$ & $46.6^{*}$ & $1 \mathrm{~J}=132.1$ & $46.2^{*}$ & $1 \mathrm{~J}=131.6$ \\
\hline $7^{\prime}-\mathrm{CH}$ & 61.9 & $1 \mathrm{~J}=137.1$ & 60.7 & $1 \mathrm{~J}=134.5$ \\
\hline $1 " '-C$ & 137.0 & & 136.6 & \\
\hline 2"'-CH & 108.7 & ${ }^{1} \mathrm{~J}=156.5,{ }^{3} \mathrm{~J}=3 \mathrm{~J}=5.4$ & 110.7 & ${ }^{1} \mathrm{~J}=156.3$ \\
\hline $3 " '-C$ & 146.7 & & 147.4 & \\
\hline $\mathrm{OCH}_{3}$ & 56.0 & $1 \mathrm{~J}=144.8$ & 55.6 & $1 \mathrm{~J}=144.1$ \\
\hline $4 " '-C$ & 145.2 & ${ }^{3} J={ }^{3} J=6.5$ & 145.2 & \\
\hline 5"'-CH & 114.4 & ${ }^{1} J=159.6$ & 115.1 & ${ }^{1} J=157.8$ \\
\hline 6"'-CH & 119.3 & ${ }^{1} \mathrm{~J}=158.6,{ }^{3} \mathrm{~J}=3 \mathrm{~J}=5.5$ & 118.76 & ${ }^{1} \mathrm{~J}=158.7,{ }^{3} \mathrm{~J}=7.5,{ }^{3} \mathrm{~J}=4.7$ \\
\hline
\end{tabular}


In compound 4, all the methylene protons $\left(2-\mathrm{CH}_{2}, 3-\mathrm{CH}_{2}\right.$ and $\left.6-\mathrm{CH}_{2}\right)$ of the dihydrodiazepine ring are diastereotopic (Figure 4a) and the assignment is based on the values of the geminal coupling constants and confirmed by the $\left({ }^{1} \mathrm{H}-{ }^{1} \mathrm{H}\right)$ gs-COSY spectrum (Figure $4 \mathrm{~b}$ ).

a)

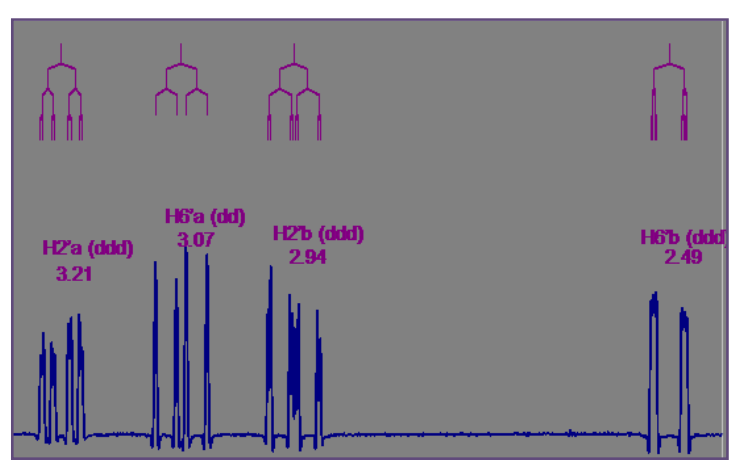

b)

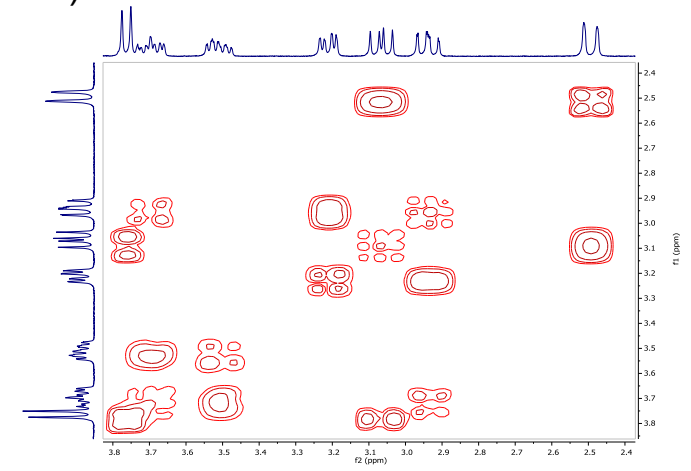

Figure 4. a) ${ }^{1} \mathrm{H}$ RMN spectrum, b) $\left({ }^{1} \mathrm{H}-{ }^{1} \mathrm{H}\right)$ gs-COSY of 4 in $\mathrm{CDCl}_{3}$.

In what concerns ${ }^{15} \mathrm{~N}$ NMR, for diazepine 3 in neutral medium we have not been able to observe the nitrogen signals and in acid media the two signals are very similar -249.5 and -251.8 ppm (Figure 5a). In the case of diazepine 4 the two nitrogen signals appear clearly differentiated, enamino at $-264.6 \mathrm{ppm}$ and amino at $-318.3 \mathrm{ppm}$ (Figure 5b).

a)

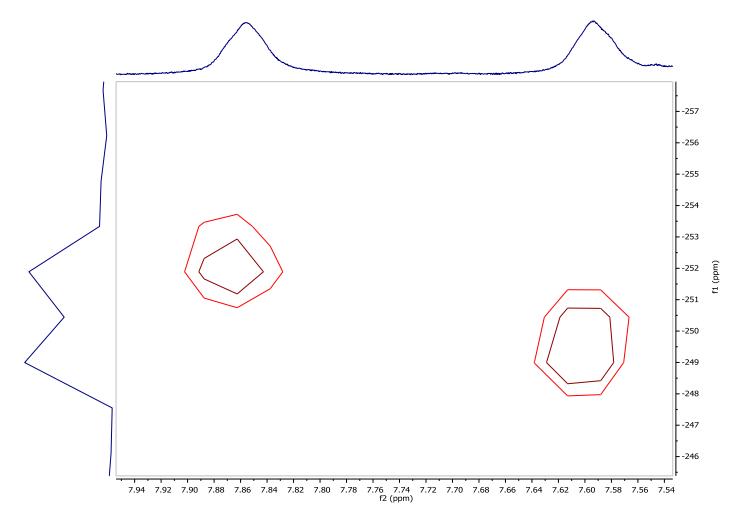

b)

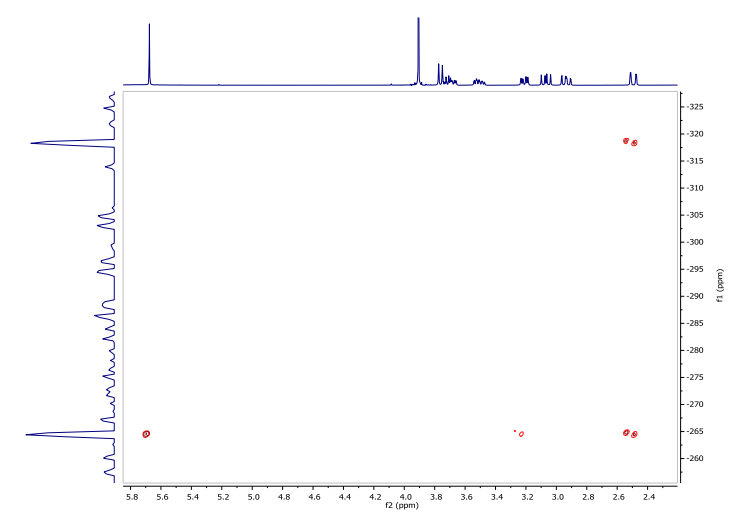

Figure a. a) ${ }^{1} \mathrm{H}-{ }^{15} \mathrm{~N}$ HMBC NMR spectrum in TFA of compound 3, b) ${ }^{1} \mathrm{H}-{ }^{15} \mathrm{~N}$ HMBC NMR spectrum of compound 4 in $\mathrm{CDCl}_{3}$.

\section{Experimental Procedure}

Solution spectra were recorded at $300 \mathrm{~K}$ on a Bruker DRX $400\left(9.4\right.$ Tesla, $400.13 \mathrm{MHz}$ for ${ }^{1} \mathrm{H}$, 100.62 $\mathrm{MHz}$ for ${ }^{13} \mathrm{C}$ and $40.56 \mathrm{MHz}$ for ${ }^{15} \mathrm{~N}$ ) spectrometer with a $5-\mathrm{mm}$ inverse detection $\mathrm{H}-\mathrm{X}$ probe equipped with a z-gradient coil for ${ }^{1} \mathrm{H},{ }^{13} \mathrm{C}$ and ${ }^{15} \mathrm{~N}$, save specified. Chemical shifts ( $\delta$ in ppm) 
are given from internal solvents, DMSO- $\mathrm{d}_{6}\left(2.49\right.$ for ${ }^{1} \mathrm{H}$ and 39.5 for $\left.{ }^{13} \mathrm{C}\right), \mathrm{CDCl}_{3}\left(7.26\right.$ for ${ }^{1} \mathrm{H}$ and 77.0 for ${ }^{13} \mathrm{C}$ ) and HMPA- $d_{18}$ (2.51 for ${ }^{1} \mathrm{H}$ and 35.8 for ${ }^{13} \mathrm{C}$ ). And external reference $\mathrm{CH}_{3}{ }^{15} \mathrm{NO}_{2}(0.00)$ for ${ }^{15} \mathrm{~N}$ NMR was used. 2D $\left({ }^{1} \mathrm{H}-{ }^{1} \mathrm{H}\right)$ gs-COSY and inverse proton detected heteronuclear shift correlation spectra, $\left({ }^{1} \mathrm{H}-{ }^{13} \mathrm{C}\right)$ gs-HMQC, $\left({ }^{1} \mathrm{H}-{ }^{13} \mathrm{C}\right)$ gs-HMBC, $\left({ }^{1} \mathrm{H}-{ }^{15} \mathrm{~N}\right)$ gs-HMQC, and $\left({ }^{1} \mathrm{H}-{ }^{15} \mathrm{~N}\right)$ gs$\mathrm{HMBC}$, were acquired and processed using standard Bruker NMR software and in non-phasesensitive mode. ${ }^{9}$ Gradient selection was achieved through a $5 \%$ sine truncated shaped pulse gradient of $1 \mathrm{~ms}$. Variable temperature experiments were recorded on the same spectrometer. A Bruker BVT3000 temperature unit was used to control the temperature of the cooling gas stream and an exchanger to achieve low temperatures.

\section{Acknowledgments}

Thanks are given to the Ministerio de Economía y Competitividad of Spain for financial support (Project number CTQ2014-56833-R).

\section{References}

1. (a) B. B. Aggarwal, A. Kumar, M. S. Aggarwal, S. Shishodia, "Curcumin Derived from Turmeric (Curcuma Longa): a Spice for All Seasons", Phytochemical in Cancer Chemoprevention, Eds. D. Bagchi and H. G. Preuss, Chapter 23, CRC Press, Boca Raton, 2005, pp. 349-387; (b) R. K. Maheshwari, A. K. Singh, J. Gaddipati, R. C. Srimal, "Multiple biological activities of curcumin: a short review", Life Sci. 2006, 78, 2081; (c) T. Esatbeyoglu, P. Huebbe, I. M. A. Ernst, D. Chin, A. E. Wagner, G. Rimbach, "Curcumin-from molecule to biological function", Angew. Chem. Int. Ed. 2012, 51, 5308; (d) K. I. Priyadarsini, "The Chemistry of Curcumin: From Extraction to Therapeutic Agent", Molecules 2014, 19, 20091; (e) M. K. Shanmugam, G. Rane, M. M. Kanchi, F. Arfyso, A. Chinnathambi, M. E. Zayed, S. A. Alharbi, B. K. H. Tan, A. P. Kumar, G. Sethi, "The Multifaceted Role of Curcumin in Cancer Prevention and Treatment", Molecules 2015, 20, 2728.

2. (a) P. Cornago, R. M. Claramunt, L. Bouissane, I. Alkorta, J. Elguero, "A study of the tautomerism of $\beta$-dicarbonyl compounds with special emphasis on curcuminoids", Tetrahedron, 2008, 64, 8089; (b) P. Cornago, P. Cabildo, D. Sanz, R. M. Claramunt, M. C. Torralba, M. R. Torres, J. Elguero, "Structure of hemicurcuminoids in the solid state and in solution", Eur. J. Org. Chem. 2013, 6043; C. I. Nieto, P. Cabildo, R. M. Claramunt, P. Cornago, D. Sanz, M. C. Torralba, M. R. Torres, M. Ferraro, I. Alkorta, J. Elguero, "The structure of $\beta$-diketones related to curcumin dtermined by X-ray crystallography, NMR (solution and solid state) and theoretical calculations", Structural Chemistry, sent for publication. 
3. (a) R. M. Claramunt, L. Bouissane, M. P. Cabildo, M. P. Cornago, J. Elguero, A. Radziwon, C. Medina, "Synthesis and biological evaluation of curcuminoid pyrazoles as new therapeutic agents in inflammatory bowel disease: effect on matrix metalloproteinases", Bioorg. Med. Chem. 2009, 17, 1290; (b) P. Cornago, P. Cabildo, R. M. Claramunt, L. Bouissane, E. Pinilla, M. R. Torres, J. Elguero, "The annular tautomerism of the curcuminoid NH-pyrazoles", New J. Chem. 2009, 33, 125; (c) C. I. Nieto, M. P. Cabildo, M. P. Cornago, D. Sanz, R. M. Claramunt, I. Alkorta, J. Elguero, J. A. García, A. López, D. Acuña-Castroviejo, "Synthesis, structure and biological activity of $3(5)$-trifluoromethyl-1Hpyrazoles derived from hemicurcuminoids", J. Mol. Struct. 2015, 1100, 518; (d) C. I. Nieto, M. P. Cabildo, M. P. Cornago, D. Sanz, R. M. Claramunt, M. C. Torralba, M. R. Torres, J. Elguero, J. A. García, A. López, D. Acuña-Castroviejo, "Fluorination Effects on NOS Inhibitory Activity of Pyrazoles Related to Curcumin", Molecules 2015, 20, 15643.

4. C. Changtam, P. Hongmanee, A. Suksamrarn, Isoxazole analogs of curcuminoids with highly potent multidrug-resistant antimycobacterial activity", Eur. J. Med. Chem. 2010, 45 4446.

5. M. C. Lozada, R. G. Enríquez, C. E. Lobato, B. Ortíz, M. Soriano, D. Gnecco, W. F. Reynolds, "Synthesis and structure of new heterocyclic derivatives of curcumin", Heterocycles 2005, 65, 49.

6. R. K. Singh, D. Rai, D. Yadav, A. Bhargava, J. Balzarini, E. De Clercq "Synthesis, antibacterial and antiviral properties of curcumin bioconjugates bearing dipeptide, fatty acids and folic acid ", Eur. J. Med. Chem. 2010, 45, 1078.

7. O. A. Hamed, N. Mehdawi, A. A. Taha, E. M. Hamed, M. A. Al-Nuri, A. S. Hussein, "Synthesis and antibacterial activity of novel curcumin derivatives containing heterocyclic moiety", Iran. J. Pharm. Res. 2013, 12, 47.

8. O. Vajragupta, P. Boonchoong, H. Watanabe, M. Tohda, N. Kummasud, Y. Sumanont, "Manganese complexes of curcumin and its derivatives: evaluation for the radical scavenging ability and neuroprotective activity", Free Radical Bio. Med., 2003, 35, 1632.

9. S. Berger, S. Braun, "200 and More NMR Experiments". Wiley-VCH, Weinheim, 2004. 